40 A PROTEIN BIOMARKER MODEL FOR DETECTION OF CARDIAC ARRHYTHMIA AND PREDICTION OF ASSOCIATED HEART FAILURE

${ }^{1} \mathrm{C}$ Tonry, ${ }^{2} \mathrm{~K}$ McDonald, ${ }^{2} \mathrm{M}$ Ledwidge, ${ }^{3} \mathrm{~B}$ Herandez, ${ }^{4} \mathrm{~N}$ Glezeva, ${ }^{3} \mathrm{C}$ Rooney, ${ }^{3} \mathrm{~B}$ Morrissey, ${ }^{3} \mathrm{~S}$ Pennington, ${ }^{3} \mathrm{~J}$ Baugh, ${ }^{1} \mathrm{C}$ Watson. ${ }^{1}$ Queens University Belfast, UK; ${ }^{2}$ STOP- HF Unit, St. Vincent's University Healthcare Group, Dublin, Ireland; ${ }^{3}$ Conway Institute University College Dublin, Ireland; ${ }^{4}$ Heartbeat Trust, Dublin, Ireland

\subsection{6/heartjil-2020-ICS.40}

Introduction Cardiac arrhythmia is strongly linked with heart failure (HF) and a primary cause of stroke. The condition affects around 37,000 people in Northern Ireland although it is estimated that many thousands more remain undiagnosed. It is important to be able to diagnose cardiac arrhythmia early, so that appropriate interventions can be made to reduce risk of subsequent stroke or HF. Currently, diagnosis and management of cardiac arrhythmia is reliant on assessment of clinical risk factors, however, routine monitoring of circulating biomarkers would significantly improve accuracy for prediction of arrhythmia and associated adverse events. The aim of this study was to (i) identify protein biomarkers, which can predict cardiac arrhythmia and (ii) identify protein biomarkers that are predictive of HF in patients with arrhythmia.

Methods Multiple Reaction Monitoring mass spectrometry-based assays were developed for measurement of a selection of candidate protein biomarkers of cardiovascular injury. Assays were developed using nanoflow reverse phase C18 chromatographic ChipCube based separation, coupled to an Agilent 6460 triple quadrupole mass spectrometer. Optimised MRM assays were applied, in a sample blinded manner, for analysis of a cohort of 410 serum samples. This included 112 patients with cardiac arrhythmia as well as matched controls without cardiac arrhythmia.

Results MRM assays were established for measurement of 25 proteins. Individually, a number of the biomarker proteins show significant differential expression between patients with and without cardiac arrhythmia. An 11-protein biomarker model was identified, which was comparable to BNP in prediction of HF within the cardiac arrhythmia subset of patients (Protein panel AUC $=0.856$ vs BNP AUC $=0.838$ ). Combination of the 11 proteins with BNP notably enhanced the predictive capacity of BNP (AUC $=0.898$ ).

Conclusions/Implications Through this study, assays have been developed for robust, multiplexed measurement of 25 cardiovascular disease-associated proteins in patient serum samples. A number of proteins were identified, which show significant expression changes in association with cardiac arrhythmia and will be further explored. Importantly, a statistical model revealed a panel of 11 proteins, which can predict $\mathrm{HF}$ in patients with cardiac arrhythmia, with comparable accuracy to BNP. This panel will need to be further validated in independent patient cohorts.

\section{A MULTI-CENTRE ANALYSIS OF THE RATES OF GUIDELINE-DIRECTED IMPLANTABLE CARDIAC DEFIBRILLATOR IMPLANTATION FOR HEART FAILURE WITH REDUCED EJECTION FRACTION: COMPARING CENTRES WITH AND WITHOUT ELECTROPHYSIOLOGY SERVICES}

${ }^{1} S$ Keane, ${ }^{2} \mathrm{R}$ Gallen, ${ }^{1} \mathrm{~T}$ Murphy, ${ }^{2} \mathrm{~J}$ Carron, ${ }^{2} \mathrm{M}$ Elmadi, ${ }^{2} \mathrm{~S}$ Quinn. ${ }^{1}$ Galway University Hospital, Galway, Ireland; ${ }^{2}$ Mater Misericordiae Hospital, Dublin, Ireland

10.1136/heartjn|-2020-ICS.41
Introduction Implantable Cardiac Defibrillators (ICD) are a potentially lifesaving therapy inserted for multiple indications. The indications for insertion of ICDs are stated in the European Society of Cardiology guidelines, and American Heart Association guidelines. One of the most common indications is for implantation in heart failure with reduced ejection fraction (HFrEF). These include patients with New York Heart Association (NYHA) Class I-III in patients with ischaemic cardiomyopathy (ICM) and NYHA Class II-III in patients with nonischaemic cardiomyopathy (NICM).

Purpose While ICDs are not exclusively implanted by electrophysiologists in the country of study, we hypothesised that a hospital having a dedicated electrophysiology service would lead to greater compliance with current guidelines.

Methods We compared two major tertiary referral centres, one of which has the largest Electrophysiology (EP) service in the country, with the other currently having no EP physician. We obtained a list of all patients who attended the respective Heart Failure Clinics for a year from the 1st of January 2018 to the 31st of December 2018. We then excluded patients with ejection fraction (EF) of $>35 \%$ by examining the relevant patients' echocardiogram reports.

Results In Heart Failure Clinic in Hospital A (with an EP service), 172 patients attending the heart failure clinic had an EF less than or equal to $35 \%$. Of those, 116 had an ICD (67.4\%); 88 with an ICD and 28 with a Cardiac Resynchronisation Therapy - Defibrillator (CRT-D). 56 patients did not have ICD/CRT. In Hospital B (without an EP service), 174 patients attending the heart failure clinic had an EF less than or equal to $35 \%$. 83 of these patients had an ICD (47.7\%). Of those, 56 had an ICD and 27 had a CRT-D. We report a significant difference of $19.7 \%(\mathrm{p}=0.0002)$ between two large tertiary referral centres in the rate of guideline-directed ICD implantation (figure 1).

Conclusion The availability of an electrophysiology service in a hospital may improve the rates of guideline-directed ICD implantation for heart failure with reduced ejection fraction. This hypothesis warrants further investigation in larger studies.

\section{A REVIEW OF MORTALITY IN PATIENTS WHO UNDERGO ICD INSERTION IN A SINGLE IMPLANTATION CENTRE}

${ }^{1} \mathrm{P}$ Wheen, ${ }^{2} \mathrm{Z}$ Sharif, ${ }^{2} \mathrm{C}$ O'Carroll-Lolait, ${ }^{3} \mathrm{D}$ Moore. ${ }^{1}$ St. James's Hospital, Dublin, Ireland: ${ }^{2}$ Beaumont Hospital, Dublin, Ireland; ${ }^{3}$ Tallaght University Dublin, Ireland

\subsection{6/heartjnl-2020-ICS.42}

Aims To review the ICD implantation rate in our institution, as well as to assess overall survival following ICD implantation, and to review the rate of early mortality $(<1$ year) following ICD insertion.

Methods Using the HeartRhythmIreland.com database, where all new ICD implants have been registered since 2005, we obtained information on demographics, implantation date, last registered visit, and notification of death. Date of death was confirmed with our own institution's electronic patient manager. Survival probability was calculated using the KaplanMeier method, using the most recent attended ICD check, or the date of death. Survival was defined as freedom from death at most recent check. (Figure 1).

Results 415 ICDs were implanted in our institution between 2005 and 2018. The mean (mean ( \pm SD)) age was 58.9 years $( \pm 14.00)$ and $333(80.2 \%)$ of the patients were 


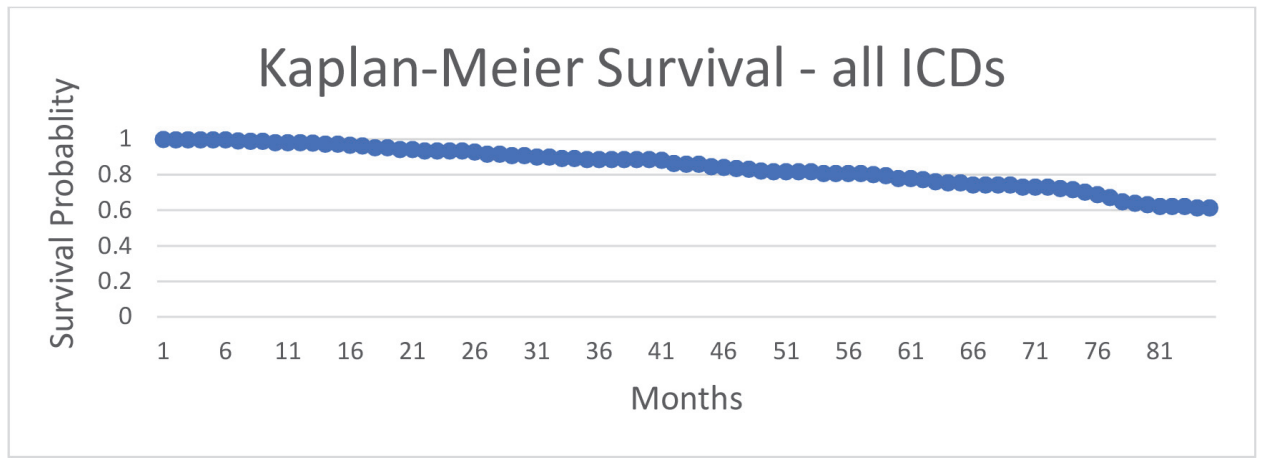

Abstract 42 Figure 1 Survival probability

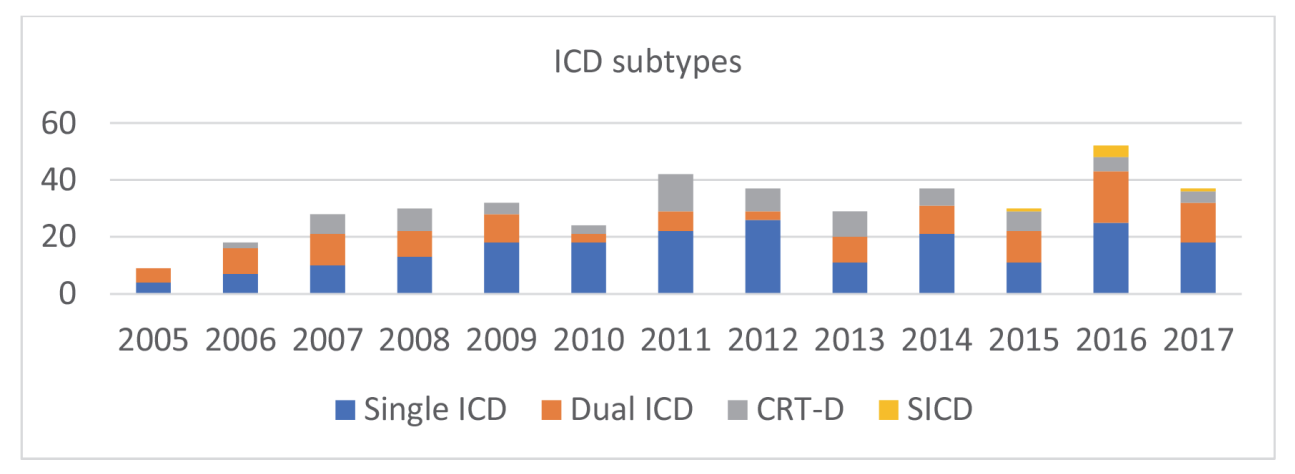

\section{Abstract 42 Figure 2 ICD Subtypes}

male. These include 80 CRT-Ds, 121 Dual chamber ICDs, 206 Single Chamber ICDs, and 7 Subcutaneous-ICDs (SICD). (Figure 2). In the case of upgrades, only the most recent device was included. 3 ICDs were explanted without replacement, 2 due to infection, and 1 due to heart transplant. 95 patients $(22.9 \%)$ with local follow up died (in whom a date of death is available in 87 patients). 4 (1.0\%) patients were lost to follow up. 6 (1.4\%) patients emigrated, and $21(5.1 \%)$ patients moved hospital within the state. Among the patients not known to have died or moved centre, the mean duration since the most recent ICD check was 4.8 months. Of the patients who died, the mean time between device insertion and death was 51.6 months \pm 30.2 , and $6(1.4 \%)$ patients died within a year of ICD insertion. Survival probability at 1,3 and 5 years was $97.9 \%, 88.7 \%$ and $79.6 \%$ respectively.

Discussion This study shows the increasing burden, and complexity, of devices being implanted within our centre. Despite our study not differentiating between primary and secondary prevention, our study shows reassuring survival probability at 5 years when compared with SCD HEF (71.1\%) and at 3 years when compared with MADIT (78\%).

\section{MICRA TRANSCATHETER PACING SYSTEM IMPLANTATION AND OUTCOMES IN A SINGLE CENTRE}

D Connellan, C Brady, P Ryan, K Walsh, J Keaney. Mater Misericordiae University Hospital, Dublin, Ireland

\subsection{6/heartjnl-2020-ICS.43}

Introduction and Objectives Traditional cardiac pacemakers incorporate transvenous leads. Recently leadless pacemakers have been approved. The Micra ${ }^{\mathrm{TM}}$ pacemaker is such a device which is inserted percutaneously via the femoral vein into the right ventricle. The device is capable of providing single chamber pacing, sensing and accelerometer based rate response. Our objective was to evaluate indications, electrical parameters at insertion, complications and follow-up of 21 patients between 2017 and 2019 who underwent Micra $^{\text {TM }}$ pacemaker insertion at our institution.

Methods This was a retrospective, observational study. All patients who had a Micra $^{\mathrm{TM}}$ pacemaker insertion in $\mathrm{MMUH}$ between 2017 and 2019 were included. Patient data was extracted from electronic hospital records.

Results 21 patients $(61.9 \%$ male $)$ had a Micra pacemaker implanted (table 1). The mean age was $42.7 \pm 17.7$ years (range, 19-81). Patient co-morbidities included cardiomyopathy (71.4\%) previous history of intracardiac lead (33.3\%), right sided valve surgery (19\%), chronic kidney disease (14.3\%), previous stroke (9.5\%), diabetes $(9.5 \%)$, and previous device infection $(9.5 \%)$. The most common underlying cardiomyopathy was congenital cardiac disease (61.9\%). 7 patients had underlying valvular heart disease $(33.3 \%)$ and 1 patient had non-ischaemic dilated cardiomyopathy.

All 21 Micra pacemaker devices were implanted successfully. $80.1 \%$ of devices were inserted into the right ventricular septum, with 2 devices inserted into the apex and the right ventricular outflow tract. 18 devices were successfully positioned on first attempt and 3 devices required repositioning (14.3\%).

There was one complication recorded. This was a pericardial effusion with tamponade, in a patient who was also undergoing lead explant. 3 patients had been lost to followup, with device checks occurring in other centres. 2 patients had died. The maximum duration of follow up was 2.9 years 